\title{
Improved Generalized-Proportionate Stepsize LMS Algorithms And Performance Analysis
}

\author{
S. C. Chan and Y. Zhou \\ Dept. of Electrical and Electronic Engineering \\ The University of Hong Kong, Hong Kong. \\ scchan@eee.hku.hk, yizhou@eee.hku.hk
}

\begin{abstract}
This paper analyzes the performance of the GPNLMS algorithm, revealing the nature of its fast convergence as well as its deficiency of inducing bigger steady state error. Based on the analysis, a class of improved GeneralizedProportionate Stepsize LMS (GPS-LMS) algorithms are proposed. With an efficient switching mechanism, the new algorithms can dynamically switch between the GP-NLMS and conventional LMS-type algorithms to achieve fast initial convergence and tracking speed and low steady state error. Computer simulations verified the superior performance of the proposed algorithms.
\end{abstract}

\section{INTRODUCTION}

Adaptive filters find important applications in signal processing, communications, control and many other areas. Many adaptive algorithms have been proposed and they can be classified broadly into two major classes: recursive least squares (RLS) and least mean squares (LMS) algorithms. Examples of LMS algorithms include the conventional LMS algorithm, the Normalized LMS (NLMS) algorithm [1], the fast LMS/Newton [2] algorithm, etc. They have a low computational complexity of $O(L)$ (where $L$ is the number of taps of the adaptive filter), which makes them attractive in those applications involving adaptive filter with high order such as Acoustic Echo Cancellation (AEC) [3]. However, the convergence speed of the conventional LMS algorithm is very sensitive to the eigenvalue spread of the input autocorrelation matrix. For highly correlated input signals, the convergence can be very low. This problem becomes more serious when the filter order increases or when the impulse response of the system to be identified is time-varying.

While the problem of highly correlated input signals can be alleviated by using transform-domain or Newton-type of adaptive filtering algorithms, the initial convergence and tracking speed of these variant LMS-type algorithms for long and sparse channel response can still be very slow. In the PNLMS algorithm first proposed by Duttweiler [4], the stepsize of each filter coefficient is adjusted in proportion to its estimated magnitude. This considerably improves the initial convergence of the NLMS algorithm. However, it still converges slowly when the impulse response is dispersive. To overcome this problem, Gay and Benesty proposed the IP-NLMS algorithm [5], where the filter coefficients are updated as a linear combination of the NLMS and PNLMS updates. These efforts have attracted considerable attention recently and this class of algorithms are usually referred to as the proportionate update algorithms $[6,7,8]$. In the Generalized Proportionate Affine Projection (GP-APA) algorithm of Hoshuyama et al [6], the step size of each filter coefficient in the APA is updated through the approximated derivatives of the filter coefficients, instead of the magnitude of the weight vector itself. Faster initial convergence and tracking speed over the conventional proportionate update algorithms were reported. When one input data vector is being processed at each iteration, it reduces to the so- called GP-NLMS algorithm, which has the lowest complexity in the GP-APA family and thus is a good alternative to other LMStype algorithms in $\mathrm{AEC}$ and other problems.

In this paper, we analyze the effect of using variable or proportionate stepsizes on the initial convergence of the LMS algorithm for white Gaussian input. It is found that the average behavior of the initial convergence can be improved if the stepsize satisfies certain ordering properties. This gives rise to a family of improved stepsize updating algorithms. We further show that the GP-NLMS algorithm satisfies this property and hence substantiate its fast initial convergence observed in extensive numerical simulation. Although the variable stepsize updating algorithm in the GP-NLMS is able to achieve a reasonably low steady state error, stepsize adaptation will unavoidably increase the mean square error (MSE) at the steady state due to additional gradient noise. This observation motivated us to propose a hybrid algorithm and an efficient switching mechanism that is able to switch from the GP-NLMS algorithm to other conventional LMS-type algorithms with a constant stepsize in order to reduce MSE when approaching the steady state. Simulation results show that this new class of improved Generalized-Proportionate Stepsize LMS (GPS-LMS) algorithms enjoys the fast initial convergence and tracking speed of the GP-NLMS algorithm and good steady state performance of other constant stepsize LMS-type algorithms. In this paper, we employed the NLMS, fast LMS/Newton and Partial Update algorithms to illustrate the good performance of the proposed algorithms. This paper is organized as follows: an analysis of the GP-NLMS algorithm is described in section II, where a new family of improved stepsize updating algorithm will be proposed. The proposed Improved GPS-LMS algorithms are presented in section III. Experimental results and comparisons are presented in section IV. Conclusions are drawn in section V.

\section{ANAL YSis of The GP-NLMS AlgorithM}

The GP-APA algorithm proposed in [6] can be written as the following GP-NLMS algorithm when one input data vector is being processed at each iteration:

$$
\begin{aligned}
& e(n)=d(n)-y(n) \\
& y(n)=W^{T}(n) \boldsymbol{X}(n), \boldsymbol{X}(n)=\left[\begin{array}{llll}
x_{1}(n) & x_{2}(n) & \cdots & x_{L}(n)
\end{array}\right]^{T} \\
& W(n+1)=W(n)+\alpha e(n) \boldsymbol{D}(n) \boldsymbol{X}(n) /\left(\boldsymbol{X}^{T}(n) \boldsymbol{D}(n) \boldsymbol{X}(n)+\delta\right) \\
& D(n)=\operatorname{diag}\left(D_{1}(n), D_{2}(n), \cdots, D_{L}(n)\right) \\
& D_{i}(n)=\frac{1-\beta}{2} \frac{\hat{c}_{i}(n-1)}{\|\hat{c}(n-1)\|_{1}}+\frac{\beta}{2 L} \\
& \|\hat{\boldsymbol{c}}(n-1)\|_{1}=\sum_{i=1}^{L}\left|\hat{c}_{i}(n-1)\right|, \hat{c}_{i}(n)=\left|w_{i}(n-1)-\hat{w}_{i}(n-1)\right| \\
& \hat{w}_{i}(n)=\eta \hat{w}_{i}(n-1)+(1-\eta) w_{i}(n-1), \quad i=1,2, \cdots, L ，
\end{aligned}
$$

where $W(n)=\left[w_{1}(n), \ldots, w_{L}(n)\right]^{T}, D_{i}(n)$ and $\hat{c}_{i}(n)$ are respectively the stepsize and approximated time derivative of the $i$-th filter tap, $\alpha$ is the global stepsize. $\beta$ serves as the minimum step size, and 
$\eta$ is the forgetting factor for calculating the smoothed tap weight $\hat{w}_{i}(n)$ and $\delta$ is a constant. $\|\cdot\|_{1}$ denotes the $l_{1}$ norm of a vector. The advantages of the GP-NLMS algorithm are two folds: First, in time-invariant channels with sparse echo path responses, the time derivatives $\hat{c}_{i}(n)$ allow significant tap weights to be given a larger stepsize and vice versa. This results in a faster initial converging speed. Secondly, since $\hat{c}_{i}(n)$ tends to reflect the time variations of the filter weights, it yields a faster tracking speed in slowly time-varying channels. We now proposed a family of improved stepsize updating algorithms and analyze the performance of the GP-NLMS algorithm.

\section{A. Improved Stepsize Updating Algorithms}

Consider the following general stepsize update scheme, which can be employed for a number of variable stepsize LMS algorithms such as the GP-NLMS:

$$
W(n+1)=W(n)+\mu e(n) D(n) X(n)
$$

where $\mu$ is the global stepsize parameter and $\boldsymbol{D}(n)=\operatorname{diag}\left(D_{1}(n), D_{2}(n), \cdots, D_{L}(n)\right)$ is a diagonal stepsize matrix, whose entities control the relative magnitude of the stepsizes for each filter coefficient. Assume that the desired signal is given by $d(n)=\boldsymbol{W}_{0}^{T} \boldsymbol{X}(n)+\eta(n)$, where $W_{0}$ is the optimal weight vector and $\eta(n)$ is a zero-mean white Gaussian additive noise which is uncorrelated with $\boldsymbol{W}(n)$ and $\boldsymbol{X}(n)$. Define the error weight vector by $\bar{V}(n)=W(n)-W_{0}$. Using (8) and the independent assumption [9], one gets

$$
\boldsymbol{V}(n+1)=\left(\boldsymbol{I}-2 \mu \boldsymbol{D}(n) \boldsymbol{R}_{X X}\right) \boldsymbol{V}(n),
$$

where $\boldsymbol{R}_{X X}$ is the autocorrelation matrix of the input vector and $\boldsymbol{V}(n)=E[\overline{\boldsymbol{V}}(n)]=E\left[\boldsymbol{W}(n)-\boldsymbol{W}_{0}\right]$ is the mean error weight vector of the variable stepsize LMS algorithms. Here, we have assume that sufficient smoothing is carried out in the stepsize update algorithm such that $D(n)$ is uncorrelated with the input and error weight vector. For simplicity, we shall assume that the input is white Gaussian distributed with $\boldsymbol{R}_{X X}=\boldsymbol{I}$. This also applies to color input when it undergoes a whitening transformation. It gives

$$
\boldsymbol{V}^{(D)}(n+1)=(\boldsymbol{I}-2 \mu \boldsymbol{D}(n)) \cdot \boldsymbol{V}(n) .
$$

The constant stepsize updating corresponds to $D(n)=I$ for all $n$. To compare the performance of the two updates, we rewrite $V^{(D)}(n+1)$ as

$$
\boldsymbol{V}^{(I)}(n+1)=\boldsymbol{V}^{(D)}(n+1)+2 \mu(\boldsymbol{D}(n)-\boldsymbol{I}) \boldsymbol{V}(n)
$$

Taking the norm of the vector, we have

$$
\begin{aligned}
\left\|\boldsymbol{V}^{(I)}(n+1)\right\|_{2}^{2} & =\left\|\boldsymbol{V}^{(D)}(n+1)\right\|_{2}^{2}+4 \mu^{2} \Sigma_{i=1}^{L}\left(D_{i}(n)-1\right)^{2} V_{i}^{2}(n) \\
& +4 \mu \Sigma_{i=1}^{L}\left(1-2 \mu D_{i}(n)\right) \cdot\left(D_{i}(n)-1\right) V_{i}^{2}(n),
\end{aligned}
$$

where $V_{i}(n)$ is the $i$-th entry in $V(n)$. Since the first two terms are positive, we only need to consider the third term as follows:

$$
\left.t_{3}=\sum_{i=1}^{L}\left[D_{i}(n)-2 \mu D_{i}^{2}(n)-1+2 \mu D_{i}(n)\right)\right] V_{i}^{2}(n)
$$

Further, assuming that $\mu$ is small compared to the other terms, we have $t_{3} \approx \sum_{i=1}^{L}\left[D_{i}(n)-1\right] V_{i}^{2}(n)$. If $D_{i}(n)$ is chosen in such a way that the rate of decrease at each time instant is proportional to the magnitude of $V_{i}(n)$, then $D_{i}(n)$ has the same order as

$\left|V_{i}(n)\right|$. Using the Chebyshev inequality, we have

$$
L \cdot \sum_{i=1}^{L}\left[D_{i}(n)-1\right] V_{i}^{2}(n) \geq \sum_{i=1}^{L}\left(D_{i}(n)-1\right) \cdot \sum_{i=1}^{L} V_{i}^{2}(n)=0,
$$

if $\sum_{i=1}^{L} D_{i}(n)=L$. This tells us that if the sum of the total stepsizes in all direction is maintained, then that new update will at least be as good as the constant stepsize update. This characterizes a class of updating schemes that can perform better than the conventional constant stepsize updates. Note, $D_{i}(n)$ is proportional to the magnitude of the corresponding entry in the weight error vector, but not the magnitude of $W_{0}$, unlike traditional proportionate stepsize LMS algorithm. The reason why convergence speeding up is observed initially in most PNLMS algorithms is due to the fact that $W(n)$ is usually initialized to the zero vector. In this case, the magnitude of $V_{i}(n)$ and $W_{0, i}$, the $i$-th entry of $W_{0}$, is identical initially. As $n$ increases, the speeding up will level off. We now analyze the stepsize updating scheme in GP-NLMS and show that it satisfies the above ordering condition.

\section{B. GP-NLMS Algorithm Stepsize Analysis}

For the GP-NLMS algorithm, we have

$\boldsymbol{c}(n+1) \approx E[\boldsymbol{W}(n+1)-\boldsymbol{W}(n)]|=| \boldsymbol{V}(n+1)-\boldsymbol{V}(n)|=| 2 \mu \boldsymbol{D}(n) \cdot \boldsymbol{U}(n) \mid$

For simplicity, assume that the input is white (say after appropriate transformation), i.e. $U(n)=V(n)$. Initially, $D(n)$ should be equal to $I$ and our stepsize estimate for the $i$-th coefficient at time instant $n+1$ is proportional to $\left|2 \mu D_{i}(n) V_{i}(n)\right|$. Hence

$$
D_{i}(n+1) \approx L \cdot \frac{\left|D_{i}(n) V_{i}(n)\right|}{\sum_{i=1}^{L}\left|D_{i}(n) V_{i}(n)\right|}=L \cdot \frac{\left|c_{i}(n+1)\right|}{\sum_{i=1}^{L}\left|c_{i}(n+1)\right|} .
$$

The factor $L$ ensures that the sum of the stepsizes is maintained. Let $V_{i}^{G P}(n)=\prod_{j=1}^{n} V_{i}(j)$, then it can be shown by induction that

$$
D_{i}(n+1) \approx L \cdot \frac{\left|V_{i}^{G P}(n)\right|}{\sum_{i=1}^{L}\left|V_{i}^{G P}(n)\right|},
$$

since $D_{i}(n+2) \approx L \cdot \frac{\left|V_{i}^{G P}(n) V_{i}(n+1)\right|}{\sum_{i=1}^{L}\left|V_{i}^{G P}(n) V_{i}(n+1)\right|}=L \cdot \frac{\left|V_{i}^{G P}(n+1)\right|}{\sum_{i=1}^{L}\left|V_{i}^{G P}(n+1)\right|}$ Finally, to stabilize the update, a hybrid update between proportionate stepsize and constant stepsize is usually employed and it gives the final update for the GP-NLMS algorithm:

$$
\mu D_{i}(n)=\frac{\mu^{\prime}}{2 L}\left[\beta \frac{L \cdot\left|c_{i}(n+1)\right|}{\sum_{i=1}^{L}\left|c_{i}(n+1)\right|}+(1-\beta)\right]=\left[\beta\left(\frac{\mu^{\prime}}{2}\right) \frac{L \cdot\left|c_{i}(n+1)\right|}{\sum_{i=1}^{L}\left|c_{i}(n+1)\right|}+(1-\beta)\left(\frac{\mu^{\prime}}{2 L}\right)\right]
$$

Since the above update satisfies the ordering property in section $A$, the mean convergence rate will be improved. As $n$ tends to infinity, $c_{i}(n)$ will tend to $(1 / L)$. However, due to the gradient noise, it will fluctuate around this value with a variance depending on the steady state MSE. This will unavoidably increase the mean square error (MSE) at the steady state due to additional gradient noise. This observation motivated us to propose in the next section a hybrid algorithm and an efficient switching mechanism that is able to switch from the GP-NLMS algorithm to other conventional LMS-type algorithms with a constant stepsize in order to reduce MSE when approaching the steady state.

\section{ThE IMPROVED GPS-LMS ALGORITHMS}

Motivated by the above analysis, it is desirable to employ the GP-NLMS algorithm during initial convergence and time-varying environments, while employing a LMS-type algorithm with a constant stepsize near the steady state in order to achieve a low 
steady state error and faster overall initial convergence and tracking speed. The key is to develop an efficient and reliable switching mechanism to switch alternately between the GP-NLMS and the constant stepsize modes according to a certain measure.

We now propose a measure based on $\hat{c}(n)$ in (6), which is similar to the one we have proposed in [8]. We found that the $l_{1}$ norm of the vector $\hat{c}(n)$ will decrease and converge gradually from its initial value to a very small value when the algorithm is about to converge to its steady state. However, this value is rather unstable during tracking of time-varying channel impulse responses. Therefore, we propose another measure to determine the switching decision between the GP-NLMS and LMS-type algorithm updates. More precisely, we compute the absolute value of the approximate derivative of $\|\hat{c}(n-1)\|_{1}$ as $G_{c}(n)=\|\hat{c}(n)\|_{1}-\|\hat{c}(n-1)\|_{1} \|_{\text {and }}$ and decaying ratio $\chi(n)=G_{c}(n) / G_{c}^{0}$, where $G_{c}^{0}$ represents the approximation of the initial level of $G_{c}(n)$, which is obtained by averaging $G_{c}(k)$ from $k=1,2, \ldots, P$. It provides a reference to measure the decay of the coefficients weight vector. A small value of $\chi(n)$ indicates a diminished variations in the weight vector, and the filter is likely to be near the end of its initial converging period. By choosing appropriately a threshold, say $\hat{\chi}$, it is possible to compare $\chi(n)$ against this threshold to determine whether switching is necessary. When $\chi(n)$ is larger than $\hat{\chi}$, the algorithm is likely to be in its initial convergence stage or in the tracking stage of a time-varying channel. The GP-NLMS update part will be chosen to give a fast initial convergence and tracking performance. On the other hand, when $\chi(n)$ falls below $\hat{\chi}$, the algorithm is likely to converge to its steady state and the LMS-type algorithms should be invoked to further lower the steady state error. To guarantee that $\chi(n)$ has actually decreased below the threshold, the switching decision should be made if $\chi(n)$ is less than the threshold for $Q$ consecutive observations, where $Q$ denotes the decision window length. The parameters $\hat{\chi}, P$ and $Q$ can be chosen experimentally in practical applications and simulation results show that the performance of the proposed algorithm is not very sensitive to these values if they are reasonably chosen.

Two constant stepsize LMS-type algorithms namely the NLMS and the fast LMS/Newton algorithms are evaluated in this work. Although the LMS part of the fast LMS/algorithm is identical to the NLMS algorithm, it works with the whitened input $\boldsymbol{u}_{a}(n)$, which requires a very small computation overhead of $6 \mathrm{M}$ additions and $6 M$ multiplications per iteration for computing the $M$-th order $\mathrm{AR}$ model of the input. Besides, a partial update (PU) scheme is employed in the GP-NLMS stepsize update to reduce its computational complexity. More specifically, when the GP-NLMS mode is active, at each odd (even) update time instant, only the stepsizes of the odd (even) coefficients are updated and the rest remain unchanged. This alternating partial update saves half of the computation cost in updating the weight vector coefficients, at the expense of mild degradation of initial convergence speed. The details of the GPS-NLMS, GPS-fast LMS/Newton and the PUGPS-NLMS algorithms are summarized in Table 1, where for simplicity, the calculation of $G_{c}^{0}$, and the comparison of $\chi(n)$ and $\hat{\chi}$ at every iteration are not included, as their computational costs are relatively low compared with the others. It can be seen from Table 1 that when the NLMS or fast LMS/Newton coefficients weight update is invoked, the conventional NLMS update or the
TABIE I

THE GENERALIZED-PROPORTIONATE STEPSIZE LMS ALGORITHMS

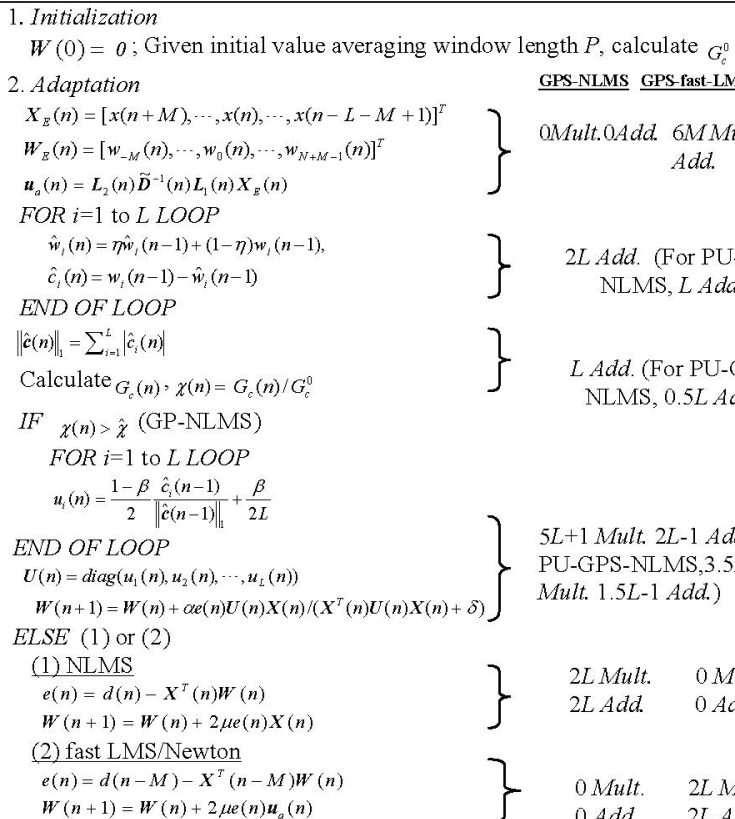

Total: GPS-NLMS (with PU): $5 L+1$ (3.5L+1) Mult. $5 L-1$ (3L-1) Add. working with GP-NLMS; $2 L(2 L)$ Mult. $5 L(3.5 L)$ Add. working with NLMS. GPS-fast LMS/Newton: $5 L+6 M+1 M u l t .5 L+6 M$-1Add. working with GPNLMS; $2 L+6 M M u l t$., $5 L+6 M A d d$. working with fast LMS/Newton.

update (9) (10) in [2] based on the whitened input $\boldsymbol{u}_{a}(n)$ will be used. Otherwise, the GP-NLMS update in (1)-(7) will be employed. Because of this sharing, the computational complexity of the proposed GPS-NLMS and GPS-fast LMS/Newton algorithm employing the original GP-NLMS scheme is at most the same as the GP-NLMS algorithm, except for the latter where a low order AR estimation part is added. Besides, this computational complexity is only required in the initial converging stage or tracking environment. At the later converging stage, the dominant updating part will be handed over to the NLMS or fast LMS/Newton algorithms which have very low arithmetic complexity.

\section{Simulation Results}

We evaluate the convergence and tracking performance of the proposed algorithms through simulations of an echo canceller for sparse channels. 100 Monte Carlo experiments and time averaging were conducted to obtain the learning curves. The system model is similar to the one used in [3], where the input signal $x(n)$ is modeled as a speech signal using an AR process with coefficients[1 $-0.650 .693-0.22 \quad 0.309-0.177]$ as given in [2]. We first examine the influence of using different values of $\beta$ on the convergence performance when the channel is a randomly generated and normalized impulse response with 28 taps. The background noise $\eta_{0}(n)$ is a zero-mean white Gaussian random sequence with variance $\delta_{\eta_{0}}^{2}(n)=0.0001$. Fig. 1 shows the MSE of running the GP-NLMS algorithm. It can be seen the use of $\beta$ results in a higher steady state MSE, due to increased gradient noise from the stepsize adaptation as discussed in Section II. B.

Next, the 128 tap echo path in the ITU-T recommendation G.168 [10] is considered. The other settings remain the same. For 
the GP-NLMS algorithm, $\eta=0.9999, \gamma=1, \alpha=1, \delta=0.00001$, and $\beta=0.5$. For the NLMS, fast LMS/Newton and the PNLMS algorithms, the stepsizes were chosen so that the steady state MSE of all the algorithms is approximately $-40 \mathrm{~dB}$. For the switching mechanism, $\hat{\chi}=0.5, P=20$, and $Q=100$. Five algorithms, the fast LMS/Newton, PNLMS, GP-NLMS and the proposed (PU-) GPS-NLMS and GPS-fast LMS/Newton algorithms were compared in two experiments. Experiment 1: Sparse echo path. From Fig. 2, we can see the proposed GPS-NLMS and GPS-fast LMS/Newton algorithms were respectively switched from the GPNLMS part to NLMS and the fast LMS/Newton part at around the $1000^{\text {th }}$ iteration. With the latter converging slightly faster than the former, these two algorithms both exhibit significantly improved overall convergence behavior and outperform the GP-NLMS in steady state MSE. The PU-GPS-NLMS algorithm was switched to the NLMS part from the GP-NLMS part at around the $1400^{\text {th }}$ iteration and converged slower than the former two though it also reached the expected low steady state MSE. Experiment 2: Tracking in sparse echo path environment. The slowly varying echo path follows the model given in [2] as $w_{i}(n+1)=w_{i}(n)+\varepsilon\left|w_{i}(n)\right| v_{i}(n), i=1,2, \cdots, L$, where $\varepsilon$ is a small constant equal to 0.015 and $v_{i}(n)^{\prime} s$ are a set of independent Gaussian white noise sequences with unit variance. The performance index is the sum of squared coefficient error (MSD). Fig. 3(a) shows the tracking performance of all the algorithms and Fig. 3(b) magnifies the learning curves between the $1700^{\text {th }}$ and the $2000^{\text {th }}$ time instants for the purpose of illustration. The GPSNLMS and GPS-fast LMS/Newton algorithms outperform the other algorithms with the fast LMS/Newton algorithm being the slowest one. Because the value of $G_{c}(n)$ varied dramatically, the proposed algorithms are working almost entirely in the GP-NLMS mode. As a result, they have the performance similar to the GPNLMS algorithm and their learning curves are nearly overlapped. However, since the PU-GPS-NLMS algorithm employs partial update to reduce the arithmetic complexity, its performance is slightly inferior to its full update counterpart. In spite of this fact, it still has the comparable performance with the GP-NLMS algorithm.

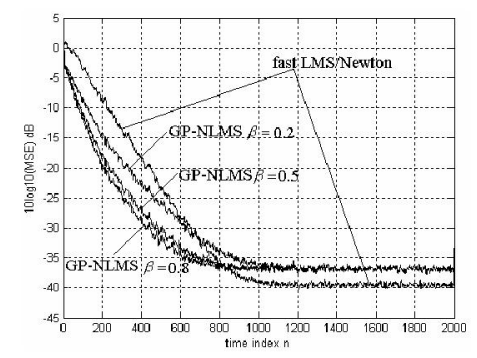

Figure 1. Influence of $\beta$ on MSE of GP-NLMS algorithm

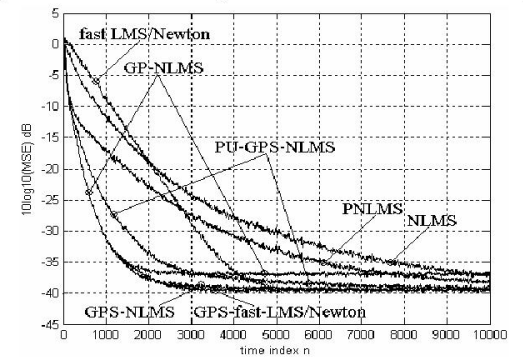

Figure 2. Example of sparse echo path, MSE vs. time.

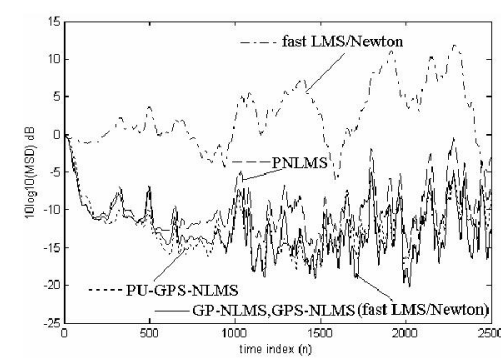

(a)

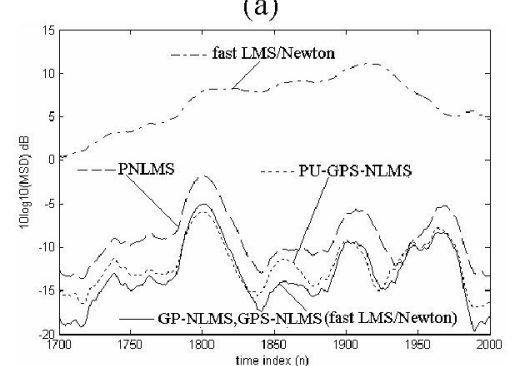

(b)

Figure 3. Tracking performance. MSD vs. time.

\section{CONCLUSion}

This paper studies the performance analysis of the GP-NLMS algorithm and presents a class of adaptive filtering algorithms called GPS-LMS algorithms. With an efficient switching mechanism, the proposed algorithms can dynamically switches between the GPNLMS algorithm and the LMS-type algorithms so that both fast initial convergence and tracking speed as well as a low steady state error can be achieved. The superior performances of the GPS-LMS algorithms are verified using computer simulations of echo cancellation problem.

\section{REFERENCES}

[1] S. Haykin, Adaptive Filter Theory, $4^{\text {th }}$ ed. Prentice Hall, 2001.

[2] B. F. Boroujeny, "Fast LMS/Newton algorithms based on autoregressive modeling and their application to acoustic echo cancellation," IEEE Trans. On Signal Processing, vol. 45, pp. $1987-$ 2000, Aug. 1997.

[3] J. Benesty et al. Advances in Network and Acoustic Echo Cancellation, Springer, 2001.

[4] D. L. Duttweiler, "Proportionate normalized least-mean-squares adaptation in echo cancelers," IEEE Trans. On Speech and Audio Processing, vol. 8, pp. 508-518, Sept. 2000.

[5] J. Benesty and S. L. Gay, "An improved PNLMS algorithm," IEEE ICASSP-02, vol. 2, pp. 1881-1884, 2002.

[6] O. Hoshuyama, R. A. Goubran and A. Sugiyama, "A generalized propotionate variable step-size algorithm for fast changing acoustic environments," IEEE ICASSP-04, vol. 4, pp. 161-164, May 2004.

[7] R. K. Martin, W. A. Sethares, R. C. Williamson and C. R. Johnson, Jr., "Exploiting sparsity in adaptive filters," IEEE Trans. On Signal Processing, vol. 50, no. 8, pp. 1883-1894, Aug. 2002.

[8] Y. Zhou, S. C. Chan and K. L. Ho, "A new proportionate fast LMS/Newton algorithm for adaptive filtering", Proc. of IEEE workshop on Statistical Signal Processing 2005, Bordeaux, France.

[9] B. Widrow, J. McCool, M. G. Larimore, and C. R. Johnson, Jr., "Stationary and nonstationary learning characteristics of the LMS adaptive filter," Proc. IEEE, vol. 64, pp. 1151-1162, Aug. 1976.

[10] "Digital Network Echo Cancellers," ITU-T Recommendation G.168, 2000. 